\title{
Avaliação econômica das associações fixas de prostaglandina/prostamida e timolol no tratamento do glaucoma e da hipertensão ocular
}

\author{
Economic evaluation of Prostaglandin/Prostamide \\ and timolol fixed combinations in the treatment \\ of glaucoma and ocular hypertension.
}

Ricardo Augusto Paletta Guedes ${ }^{1}$, Vanessa Maria Paletta Guedes², Julia Linhares Borges ${ }^{3}$, Alfredo Chaoubah ${ }^{4}$

\section{ResUmo}

Objetivo: Avaliar o custo ao final de 5 anos, a efetividade e a relação custo-efetividade das associações fixas de prostaglandina ou prostamida com timolol $0,5 \%$ para o tratamento do glaucoma e da hipertensão ocular no Estado de Minas Gerais, Brasil. Métodos: Este estudo transversal avaliou as seguintes associações fixas: bimatoprosta/timolol 0,5\% (BT), latanoprosta/timolol 0,5\% (LT) e travoprosta/timolol 0,5\% (TT). O custo foi calculado a partir do número médio de gotas de 5 frascos de cada associação, da duração (dias) e do preço máximo ao consumidor (PMC). A efetividade na redução da pressão intraocular (PIO) foi obtida na literatura. Para cada uma das associações, calculou-se o custo diário, mensal, anual e em 5 anos. A relação custo-efetividade foi definida como o custo em 5 anos de cada percentual de redução da PIO. Resultados: O PMC, número médio de gotas por frasco e a duração média (dias) foram, respectivamente: $R \$ 83,07 ; 109,4$ e 54,7 para BT; $R$ \$ 126,$03 ; 97,0$ e 48,5 para LT e R $\$ 97,47 ; 96$ e 48,0 para TT.A capacidade de redução percentual da PIO encontrada na literatura foi $35,10 \%$ para BT,35,00\% para LT e $34,70 \%$ para TT. O custo em 5 anos para cada percentual de redução da PIO foi de R $\$ 61,02$ para BT, R $\$ 104,71$ para LT e R \$ 82,53 para TT.A associação BT é dominante sobre as demais. Conclusões: BT apresentou em 5 anos menor custo e maior efetividade que LT e TT.

Descritores: Glaucoma/economia; Glaucoma/quimioterapia; Pressão intraocular/economia; Pressão intraocular/quimioterapia; Anti-hipertensivos/economia; Antihipertensivos/administração \& dosagem; Timolol/administração \& dosagem;Timolol/ economia; Prostaglandinas F sintéticas/administração \& dosagem; Prostaglandinas F sintéticas/economia; Combinação de medicamentos; Análise custo-efetividade

\footnotetext{
${ }^{1}$ Mestre em Saúde Coletiva; Especialista em glaucoma pelo Hospital des Quinze-Vingts (Paris, França); Pesquisador da Universidade Federal de Juiz de Fora - UFJF - Juiz de Fora (MG), Brasil;

${ }^{2}$ Especialista em glaucoma pelo Hospital des Quinze-Vingts (Paris, França); Pesquisadora da Universidade Federal de Juiz de Fora UFJF - Juiz de Fora (MG), Brasil;

${ }^{3}$ Acadêmica da Faculdade de Medicina da Universidade Federal de Juiz de Fora (UFJF);

${ }^{4}$ Doutor, Professor do Departamento de Estatística e Pesquisador da Universidade Federal de Juiz de Fora - UFJF - Juiz de Fora (MG), Brasil Instituição onde o estudo foi realizado: Universidade Federal de Juiz de Fora.
}

Os autores afirmam a inexistência de conflitos de interesse

Recebido para publicação em: 9/11/2009 - Aceito para publicação em 27/6/2010 


\section{INTRODUÇÃO}

$\mathbf{O}$ tratamento da hipertensão ocular e do glaucoma consiste na redução da pressão intraocular (PIO) a níveis que impeçam o aparecimento ou a progressão de neuropatia óptica (PIOalvo). A redução da PIO pode ser realizada através de medicamentos, laser ou cirurgia. ${ }^{(1)}$

A terapia inicial do glaucoma e da hipertensão ocular é geralmente feita através do uso de colírios em monoterapia. Caso haja uma redução suficiente da PIO, com uma única medicação, aconselham-se controles periódicos. Segundo o Consenso da Sociedade Brasileira de Glaucoma (SBG), nos casos em que a PIO-alvo não foi atingida com uma medicação e houve pelo menos $10 \%$ de redução da PIO, uma segunda droga deverá ser adicionada à primeira. ${ }^{(1,2)}$ Dados do Ocular Hypertension Treatment Study (OHTS) mostram que quase metade dos hipertensos oculares necessitaram 2 ou mais drogas para atingirem a PIO-alvo. ${ }^{(3)}$ Já no Early Manifest Glaucoma Trial (EMGT), aproximadamente $70 \%$ dos portadores de glaucoma inicial usavam 2 ou mais medicamentos para atingirem a PIOalvo. ${ }^{(4)}$ Quanto mais avançado o glaucoma, um maior número de colírios é necessário. Em uma avaliação multicêntrica dos custos relacionados ao glaucoma na Europa, a média mensal de colírios por paciente variou de 1,1 nos casos iniciais a 2,4 nos casos mais avançados. ${ }^{(5)}$

As associações fixas de análogos de prostaglandinas ou prostamida com o maleato de timolol a $0,5 \%$ foram recentemente introduzidas como opções terapêuticas válidas, trazendo diversas vantagens. São colírios com duas medicações de efeitos complementares em um mesmo frasco. $\mathrm{O}$ maleato de timolol a $0,5 \%$ reduza produção do humor aquoso, enquanto que os análogos de prostaglandina/prostamida, aumentam o escoamento pela via não convencional. Por serem utilizados uma vez ao dia, facilitam o regime terapêutico, evitam o efeito wash-out, proporcionando uma melhora da aderência e eficácia do tratamento. ${ }^{(6)}$

O objetivo deste estudo de fármaco-economia foi uma análise de custo-efetividade das seguintes associações fixas para o tratamento do glaucoma e da hipertensão ocular: bimatoprosta $0,03 \% /$ timolol $0,5 \%$ (BT; Ganfort, Allergan, Inc, Irvine, CA, EUA); latanoprosta 0,005\%/timolol 0,5\% (LT, Xalacom, Pfizer, New York, NY,EUA) e travoprosta 0,004\%/timolol 0,5\% (TT, DuoTravatan, Alcon, Fort Worth, TX, EUA).

\section{Métodos}

Este estudo transversal avaliou o preço máximo ao consumidor, no mês de novembro de 2009, (alíquota do Imposto sobre Circulação de Mercadorias e Serviços,
ICMS, de $18 \%$ para o Estado de Minas Gerais) das diferentes apresentações farmacológicas das seguintes associações fixas: BT, LT e TT disponíveis no mercado em relação a sua efetividade.

Os dados clínicos referentes à efetividade das medicações estudadas foram obtidos do estudo de Hommer et al. ${ }^{(7)}$ Estes autores realizaram uma revisão sistemática da literatura de ensaios clínicos randomizados, envolvendo as associações fixas supracitadas.

O custo das medicações foi obtido do guia das farmácias (mês de novembro de 2009) através do preço máximo ao consumidor. Os autores calcularam o número médio de gotas no frasco de cada uma das medicações estudadas. Este cálculo foi realizado da seguinte maneira: foi feita uma contagem do número de gotas em 5 frascos de cada associação fixa (BT, LT e TT). Os frascos foram segurados com o conta-gotas para baixo em uma angulação de $90^{\circ}$ pela mesma pessoa. Após a contagem das gotas, obteve-se a média e o desvio padrão para cada associação fixa estudada.

Uma vez obtido o número médio de gotas no frasco de cada associação fixa, calculou-se a duração média do frasco, o custo diário, mensal, anual e em 5 anos.

A relação custo-efetividade foi definida como o custo em 5 anos para cada unidade percentual de redução da PIO, sendo calculada pela divisão do custo em 5 anos de cada associação fixa por sua respectiva efetividade (medida em percentual de redução da PIO). Calculou-se ainda a razão de custo-efetividade incremental (ICER). Seguindo as diretrizes para estudos econômicos do Ministério da Saúde brasileiro, foi aplicada uma taxa de desconto de $5 \% .{ }^{(8)}$

A robustez dos resultados de análise de custoefetividade foi testada através de análises de sensibilidade univariadas. Diferentes taxas de descontos $(0$ e $10 \%$ ), bem como mudanças no custo das associações fixas e da duração do frasco (20\% a mais em casos de baixa aderência ou duração fixa de 30 dias para os casos de desperdício de gotas) foram avaliadas.

Todos os cálculos foram feitos usando-se Excel 2007 (Microsoft).

\section{Resultados}

O preço máximo ao consumidor, no mês de novembro de 2009, das medicações estudadas foi o seguinte: $\mathrm{R} \$ 83,07$ para a associação $\mathrm{BT}, \mathrm{R} \$ 126,03$ para a associação LT e R $\$ 97,47$ para a associação TT.

A Tabela 1 mostra o número de gotas obtido na contagem dos frascos, bem como o número médio de 
Tabela 1

Número de gotas obtido na contagem dos frascos e o número médio de gotas e o desvio padrão para cada associação fixa

\begin{tabular}{cccccccc}
\hline Medicação & Frasco 1 & Frasco 2 & Frasco 3 & Frasco 4 & Frasco 5 & Média & DP \\
\hline BT & 110 & 111 & 110 & 108 & 108 & 109,4 & 1,3 \\
LT & 99 & 102 & 94 & 95 & 95 & 97,0 & 3,4 \\
TT & 100 & 95 & 95 & 94 & 96 & 96,0 & 2,3 \\
\hline
\end{tabular}

BT- associação fixa Bimatoprosta 0,03\%/Timolol 0,5\%; LT- associação fixa Latanoprosta $0,005 \%$ / Timolol 0,5\%; TT- associação fixa Travoprosta 0,004\%/Timolol 0,5\%; DP- desvio padrão

Tabela 2

\begin{tabular}{cccccc}
\multicolumn{5}{c}{$\begin{array}{c}\text { Duração média do frasco (admitindo-se } \mathbf{1} \text { gota em cada olho por dia sem } \\
\text { desperdício ou esquecimento), o custo diário, mensal, anual e em } \mathbf{5} \text { anos para } \\
\text { cada associação fixa de medicações (preços em reais) }\end{array}$} \\
Medicações & $\begin{array}{c}\text { Duração } \\
\text { média } \\
\text { (dias) }\end{array}$ & $\begin{array}{c}\text { Custo } \\
\text { diário } \\
\text { (R\$) }\end{array}$ & $\begin{array}{c}\text { Custo } \\
\text { mensal } \\
\text { (R\$) }\end{array}$ & $\begin{array}{c}\text { Custo } \\
\text { anual } \\
\text { (R\$) }\end{array}$ & $\begin{array}{c}\text { Custo } \\
\text { em 5 anos } \\
\text { (R\$) }\end{array}$ \\
\hline BT & 54,70 & 1,52 & 45,56 & 546,71 & $2.733,56$ \\
LT & 48,50 & 2,60 & 77,96 & 935,48 & $4.677,40$ \\
TT & 48,00 & 2,03 & 60,92 & 731,03 & $3.655,13$ \\
\hline
\end{tabular}

BT- associação fixa Bimatoprosta 0,03\%/Timolol 0,5\%; LT- associação fixa Latanoprosta 0,005\%/ Timolol $0,5 \%$; TT- associação fixa Travoprosta $0,004 \%$ /Timolol $0,5 \%$.

Tabela 3

Análise de custo-efetividade das associações fixas

\begin{tabular}{|c|c|c|c|c|c|c|}
\hline Medicações & $\begin{array}{l}\text { Custo em } \\
5 \text { anos } \\
(\mathbf{R} \$)^{*}\end{array}$ & $\begin{array}{c}\text { Efetividade } \\
(\% \text { de redução da PIO) } * *\end{array}$ & $\begin{array}{c}\text { Relação } \\
\text { custo-efetividade } \\
(\mathbf{R} \$ / \%)\end{array}$ & $\begin{array}{c}\text { Custo } \\
\text { incremental } \\
(\mathbf{R} \$)\end{array}$ & $\begin{array}{c}\text { Efetividade } \\
\text { incremental } \\
(\%)\end{array}$ & $\begin{array}{c}\text { ICER } \\
(\mathbf{R} \$ / \%)\end{array}$ \\
\hline BT & $2.141,82$ & 35,10 & 61,02 & - & - & Dominante \\
\hline $\mathrm{LT}$ & $3.664,87$ & 35,00 & 104,71 & $-1.523,05$ & 0,1 & Dominado \\
\hline TT & $2.863,89$ & 34,70 & 82,53 & $-722,07$ & 0,4 & Dominado \\
\hline
\end{tabular}

BT- associação fixa Bimatoprosta 0,03\%/Timolol 0,5\%; LT- associação fixa Latanoprosta $0,005 \% /$ Timolol $0,5 \%$; TT- associação fixa Travoprosta $0,004 \%$ /Timolol 0,5\%; ICER- Razão de custo-efetividade incremental

* Custo em 5 anos com desconto de $5 \%{ }^{\left({ }^{(8)}\right.}$

** Valores obtidos de Hommer A et al. ${ }^{(7)}$

gotas e o desvio padrão para cada associação fixa. A duração do frasco, o custo diário, mensal, anual e em 5 anos para cada associação de medicações estudada está demonstrado na Tabela 2 .

A análise de custo-efetividade está apresentada na Tabela 3. O custo de cada percentual de redução da PIO ao final de 5 anos foi de R \$ 61,02 para a associação BT, R\$ 104,71 para a associação LT e R \$ 82,53 para a associação TT. A associação BT é dominante sobre as demais, pois possui uma efetividade maior com um custo menor.

$\mathrm{Na}$ análise de sensibilidade, mudando-se a taxa de desconto para $0 \%$ ou para $10 \%$, não há mudança do resultado, ou seja, a associação BT continua sendo dominante. Levando-se em consideração uma aderência de $80 \%$ para cada um dos medicamentos (ou seja, uma duração de $20 \%$ a mais de cada frasco) ou uma duração fixa de 30 dias (admitindo-se desperdício de gotas), o resultado também continua inalterado. Pela análise de sensibilidade, seria preciso abaixar o custo da associação TT em 30\% (custo de R $\$ 68,23$ por frasco) e da associação LT em 50\% (custo de R $\$ 63,02$ por frasco) para que eles se tornem uma opção custo-efetiva comparável à associação BT. 


\section{DiscussÃo}

O custo com tratamento de doenças crônicas, entre elas o glaucoma, têm um grande impacto no orçamento global da saúde. ${ }^{(9)}$ Os economistas especializados em saúde acreditam que a inovação nas tecnologias médicas (fármacos, procedimentos médicos-cirúrgicos, e equipamentos médicos) é o fator primário do aumento do custo da saúde, observado nos últimos anos. ${ }^{(10)}$

Os recursos destinados à saúde são limitados, portanto estudos de avaliação econômica ganham cada vez mais importância, por facilitar a tomada de decisões por parte dos gestores da saúde. Os principais tipos de avaliação econômica de tecnologias em saúde são estudos de custo-minimização, custo-efetividade, custo-benefício e custo-utilidade. Os estudos de custo-efetividade têm valor, pois agregam no resultado tanto o custo desta tecnologia estudada quanto o resultado que ela proporciona. ${ }^{(10)}$

As prostaglandinas (e prostamida) são consideradas, na maioria das vezes, como as medicações de primeira escolha no tratamento do glaucoma e da hipertensão ocular, graças ao perfil de segurança e eficácia. ${ }^{(1,2)}$ Guedes et al. estudaram qual seria a prostaglandina mais custo-efetiva para a população brasileira. Neste estudo, a bimatoprosta foi superior à latanoprosta e à travoprosta. ${ }^{(11)}$ Este mesmo resultado foi obtido por pesquisadores em outros locais do mundo. ${ }^{(12,13)}$

No entanto, em grande parte dos casos, faz-se necessário a adição de uma segunda droga com o objetivo de se atingir a PIO-alvo. ${ }^{(3,4)}$ As associações fixas de prostaglandina/timolol foram recentemente incorporadas no mercado mundial e brasileiro. Ao disponibilizar duas medicações de ações complementares em um mesmo frasco, elas têm a vantagem de facilitar a posologia, aumentar a aderência e reduzir a PIO de maneira eficiente. ${ }^{(6)}$ Diversos estudos mostraram a segurança clínica e a eficácia destas associações fixas para o tratamento clínico do glaucoma e da hipertensão ocular. ${ }^{(6)}$

Do ponto de vista econômico, Martinez et al., na Espanha, concluíram que a associação fixa BT seria a melhor opção. Nesse estudo, o custo de cada percentual de redução da PIO ao final de 3 meses foi de 5,34 euros para BT, 5,40 euros para LT e 5,45 euros para TT. ${ }^{(14)}$ Hommer et al. encontraram resultado semelhante ao comparar os resultados de custo-efetividade das associações fixas em 5 países europeus: Reino Unido, Suécia, Noruega, Itália e Espanha. ${ }^{(7)}$

No presente estudo, a associação fixa BT foi superior às demais quando avaliada do ponto de vista econômico. O custo para cada percentual de redução da PIO ao final de 5 anos foi de $\mathrm{R} \$ 61,02$ para BT, $\mathrm{R} \$ 104,71$ para LT e R \$ 82,53 para TT. Isto leva uma razão de custo- efetividade incremental (ICER) negativa, fazendo com que a associação BT seja dominante sobre as demais.

Algumas razões podem ser apontadas para o resultado encontrado. A associação BT possui $0,5 \mathrm{ml}$ a mais no frasco do que as demais ( $3 \mathrm{ml}$ versus $2,5 \mathrm{ml}$ ). Isto leva a uma duração maior do frasco, consequentemente um menor custo por gota. No estudo de Guedes et al. sobre a relação de custo-efetividade das prostaglandinas, observa-se que o volume do frasco tem uma grande influência no resultado de custo-efetividade (bimatoprosta em frascos de $5 \mathrm{ml}$ é mais custo-efetiva que a bimatoprosta em frascos de $3 \mathrm{ml})$. $^{(11)}$ Outra razão importante é que o preço máximo ao consumidor (PMC) da associação BT é menor que das outras $(R \$ 83,07$ versus $R \$ 126,03$ para a associação LT e R \$ 97,47 para a associação TT). A combinação dos fatores citados: conteúdo maior do frasco e PMC menor faz com que a associação BT tenha um menor custo diário, mensal, anual e em 5 anos.

Além do fato de a associação BT ser mais barata, existe ainda evidência de que ela é mais eficaz. Dados da literatura compilados e analisados por Hommer et al. mostram que a redução percentual média da PIO é de $35,1 \%$ para $\mathrm{BT}, 35,0 \%$ para $\mathrm{LT}$ e $34,7 \%$ para TT com PIO média basal variando de 25,6 a $26,0 \mathrm{mmHg} .{ }^{(7)}$ Barnebey et al. encontraram uma redução média percentual de $38,1 \%$ para a associação TT, ${ }^{(15)}$ porém a PIO basal média era de $30,2 \mathrm{mmHg}$, bem acima da médias dos outros estudos clínicos. Por esta razão, os autores do presente estudo escolheram para efetividade os valores apresentados por Hommer et al. ${ }^{(7)}$ Este estudo usa dados clínicos secundários, portanto está sujeito a limitações. Os dados de efetividade foram obtidos de estudos diferentes, pois não há ainda na literatura uma comparação direta de maneira randomizada e prospectiva entre as 3 associações fixas estudadas. A diferença observada na efetividade entre as associações fixas é muito pequena, ${ }^{(7)}$ podendo-se levantar a questão da real validade clínica desta diferença.

Outra limitação é que o cálculo do custo foi baseado no PMC, seguindo as orientações do painel para estudos de custo-efetividade. ${ }^{(16)} \mathrm{O}$ preço real de aquisição do medicamento pode ser diferente tanto do ponto de vista individual (programas de fidelização dos laboratórios ou farmácias) quanto coletivo (ao comprar grandes quantidades, gestores de sistemas de saúde conseguem preços menores).

$\mathrm{Na}$ análise de sensibilidade, o único fator que poderia alterar o resultado foi o preço.Ao se reduzir o custo da associação LT em $50 \%$ e da TT em $30 \%$ estas passam a apresentar relação de custo-efetividade semelhante. Se o custo das associações fixas (custo/gota) fosse semelhante, a relação custo-efetividade provavelmente também seria, visto que a efetividade delas mostra muito pouca diferença. 
No ambiente do Sistema Único de Saúde (SUS), os resultados de avaliações econômicas como esta podem se transformar em um importante instrumento nos processos de decisão sobre a incorporação de tecnologias e a alocação de recursos em saúde. Estas análises devem auxiliar na seleção de intervenções mais efetivas por um menor custo e agregar elementos para aprimorar políticas públicas de saúde. ${ }^{(8)}$

\section{Conclusão}

A associação BT apresentou menor custo ao final de 5 anos, assim como uma capacidade de redução percentual da PIO maior. Portanto, a associação fixa de prostaglandina ou prostamida com timolol mais custoefetiva é a associação BT.

\section{Abstract}

Purpose: To assess the 5-year cost, effectiveness and costeffectiveness of fixed combinations of prostaglandin or prostamide and timolol $0.5 \%$ on glaucoma and/or ocular hypertension in the state of Minas Gerais, Brazil. Methods: This cross-sectional study evaluated the following fixed combinations: bimatoprost/timolol 0.5\% (BT), latanoprost/timolol $0.5 \%$ (LT) and travoprost/ timolol $0.5 \%(T T)$. Cost was obtained through mean number of drops in a sample of 5 containers of each medication, duration (days) and the average wholesale price (AWP). Effectiveness in reducing intraocular pressure IOP was derived from the literature. Daily, monthly, annually and 5-year cost was calculated. Costeffectiveness was defined as cost by each percentage of $I O P$ reduction over 5 years. Results: $A W P$, mean number of drops and mean duration (days) were: $R \$ 83.07 ; 109.4$ and 54.7 for BT; $R \$ 126.03 ; 97.0$ and 48.5 for LT and $R \$$ 97.47; 96.0 and 48.0 for TT. Mean percentage of IOP reduction, obtained from literature, was: $35.10 \%$ for $B T$, $35.00 \%$ for $L T$ and $34.70 \%$ for TT. Cost-effetiveness ratio (R\$/\%) was: 61.02 for BT, 104.71 for LT and 82.53 for TT. $B T$ was dominant over LT and TT. Conclusion: BT presented lower costs and better effectiveness when compared to LT and TT. The most cost-effective fixed combination was BT.

Keywords: Glaucoma/economics; Glaucoma/drug therapy; Intraocular pressure/economics; Intraocular pressure/drug therapy; Antihypertensive agents/ administration \& dosage Antihypertensive agents/ economics; Timolol/administration \& dosage; Timolol/ economics; Prostaglandins F, Synthetic/administration \& dosage; Prostaglandins F, synthetic/economics; Drug combinations; Cost-effectiveness analysis

\section{REFERÊNCIAS}

1. European Glaucoma Society. Terminology and guidelines for glaucoma. 2nd ed. Savona: Dogma; 2003.

2. Sociedade Brasileira de Glaucoma. $2^{\circ}$ Consenso Brasileiro de Glaucoma Primário de Ângulo Aberto. São Paulo: PlanMark; 2005.

3. Kass MA, Heuer DK, Higginbotham EJ, Johnson CA, Keltner JL, Miller JP, et al. The Ocular Hypertension Treatment Study: a randomized trial determines that topical ocular hypotensive medication delays or prevents the onset of primary openangle glaucoma. Arch Ophthalmol. 2002;120(6):701-13; discussion 829-30.

4. Leske MC, Heijl A, Hussein M, Bengtsson B, Hyman L, Komaroff E; Early Manifest Glaucoma Trial Group. Factors for glaucoma progression and the effect of treatment: the early manifest glaucoma trial. Arch Ophthalmol. 2003;121(1):48-56.

5. Traverso CE, Walt JG, Kelly SP, Hommer AH, Bron AM, Denis $P$, et al. Direct costs of glaucoma and severity of the disease: a multinational long-term study of resource utilization in Europe. Br J Opthalmol. 2005;89(10):1245-9.

6. Cox JA, Mollan SP, Bankart J, Robinson R. Efficacy of antiglaucoma fixed combination therapy versus unfixed components in reducing intraocular pressure: a systematic review. Br J Ophthalmol. 2008;92(6):729-34.

7. Hommer A, Wickstrom J, Friis MM, Steeds C, Thygesen J, Ferreras A, et al. A cost-effectiveness analysis of fixed-combination therapies in patients with open-angle glaucoma: a European perspective. Curr Med Res Opin. 2008;24(4):1057-63.

8. Brasil. Ministério da Saúde. Secretaria de Ciência, Tecnologia e Insumos Estratégicos. Departamento de Ciência e Tecnologia. Diretrizes metodológicas: estudos de avaliação econômica de tecnologias em saúde. Brasília: Ministério da Saúde; 2009

9. Guedes RAP, Guedes VMP. Custo crescente em glaucoma: atualidades e seu impacto na saúde coletiva. Rev APS. 2008;11(4):444-50.

10. Rascati KL. Introdução à farmacoeconomia. Porto Alegre: Artmed; 2009.

11. Guedes RAP, Guedes VMP, Chaoubah A. Custo-efetividade dos análogos de prostaglandinas no Brasil. Rev Bras Oftalmol. 2008;67(6):281-6.

12. Frenkel RE, Frenkel M, Toler A. Pharmacoeconomic analysis of prostaglandin and prostamide therapy for patients with glaucoma or ocular hypertension. BMC Ophthalmology. 2007;7:16.

13. Noecker RJ, Walt JG. Cost-effectiveness of monotherapy treatment of glaucoma and ocular hypertension with the lipid class of medications. Am J Ophthalmol. 2006,;141(1 Suppl):S15-21.

14. Martínez A, Slof J. [Cost-efficacy analysis of fixed combinations of prostaglandin/prostamide for treating glaucoma]. Arch Soc Esp Oftalmol. 2008;83(10):595-600. Spanish.

15. Barnebey HS, Orengo-Nania S, Flowers BE, Samples J, Mallick S, Landry TA, Bergamini MV. The safety and efficacy of travoprost $0.004 \% /$ timolol $0.5 \%$ fixed combination ophthalmic solution. Am J Ophthalmol. 2005;140(1):1-7.

16. Brown GC, Brown MM, Sharma S, Brown H, Smithen L, Leeser D, Beauchamp G. Value-based medicine and ophthalmology: an appraisal of cost-utility analysis. Trans Am Ophthalmol Soc. 2004;102:177-85; discussion 185-8.

\section{Endereço para correspondência: \\ Ricardo Augusto Paletta Guedes}

Av. Rio Branco, $n^{\circ} 2337$ sls 801, 807, 808 - Centro

CEP 36010-905 - Juiz de Fora - (MG), Brasil 